\title{
The high dilution phenomenon, yours to discover!
}

\author{
Carlos Renato Zacharias
}

\author{
Editor-in-Chief, International Journal of High Dilution Research - IJHDR
}

A new year has arrived and 2009 will surely represent an important year for IJHDR. This current issue celebrates our first anniversary and we are confident in growing to new and higher standards in the editorial and scientific communities. IJHDR was born from a desire to see $\mathrm{HD}$ research rise to the level of the other classical areas. For this reason, it is constantly being adjusted to the models of the best scientific publications. Policies, guidelines, website, editorial team and many other details were revised to improve its quality. The result can be measured in our visitation map and by the first indexations we have already received. In short, IJHDR is starting this new year ready to face new challenges and to contribute to the High Dilution's Science development, dealing with a so common as mysterious phenomenon. Common, due to two centuries of clinical evidences reinforced by modern experimental multidisciplinary researches. Mysterious, while still requiring a better characterization and modeling according to the current scientific knowledge. In many aspects, HD Science is following the same steps as other fields: current experimental evidences without a well established theoretical description. What would it means?

Day counting is also a curious phenomenon. Many cultures celebrate the New Year in January, while many others in different months. The same diversity can be found for the months and years counting. Julian, Hebrew, Islamic, Chinese, Maia and many different calendars have been proposed based on experimental observations revealing natural cycles. No matter how one measure the day length, it is always the same. In fact the sunlight and moonlight periods change as the days come in. However, both periods together take the same value for all days. And the Sun-Moon daily dominance assumes a cyclical behavior after some days. All civilizations despite their cultural background differences realized that these signs were important to control the time step and modeled them accordingly their beliefs and Science, organizing those cycles in calendars. Such knowledge made them able to foresee rainy and dried seasons, the rivers' level, improve the agriculture, hunt, war and even talk with their divinities. No matter how mythological or limited, whether Solar or Lunar based, those calendars addressed to a common phenomenon: the relative astronomical movements, mainly of the Sun, Earth and Lune. Despite how different old civilizations have described the common natural cycles, trying to reveal and dominate such mysterious order, they were able to progress and survive within their limitations.

In many aspects the current $\mathrm{HD}$ community is living in a similar situation as our ancients. Maybe in a lower level yet. Although the clinical practice has given us a daily evidence of a so common as mysterious order, with phenomenological observations reported in the Materia Medica compendiums, researchers are confused about how to model and describe it. Our community is still unable to characterize the HD phenomenon, despite our expertise in all established scientific fields. All of us are invited to reflect about our current knowledge and limitations, before explore the HD phenomenon, in order to discover its secrets as well our connections with it.

Maybe the New Year of 2009 would not be new, neither a starting period, nor 2009. No matter how we have conceived our calendars and Science, and while we have been dedicating our efforts discussing personal, cultural, scientific or philosophical points of view and beliefs, Sun, Earth, Moon and many others astronomical bodies remain dancing around us, maybe waiting for our progress or claiming for our attention.

Despite calendars, 2009 was elected to celebrate the International Year of Astronomy (IYA2009) a global effort initiated by the International Astronomical Union (IAU) and UNESCO to help the citizens of the world to rediscover their place in the Universe through the day- and night-time sky, and thereby engage a personal sense of wonder and discovery. The official slogan of the IYA2009 is "The Universe, Yours to Discover" (http://www.astronomy2009.org/general/).

HD community is not yet globally organized to propose a similar effort to help the researchers challenge their limitations, engaging all in a 
collective task force to reveal the HD mysteries. However this moment is coming, as we are already locally organized in several centers of the world.

IJHDR has been running in this direction, as an international, online and free vehicle of Science dissemination dedicated to HD research. For this current issue, we have selected some articles to stimulate our thoughts. While a NMR study keeps alive the hypothesis for structural solvent arrangement due to dynamization procedure, an alternative broiler production management contributes to reflect about a sustainable animal technology. A clinical report reinforces the possibilities of the homeopathic treatment and a reflection about chemical constitutional remedies is presented. Finally, an inflammatory model testing HD from copaiba oil help us to reflect about technological use for HD, as well to think about its action mechanisms.

Let's enjoy us, all together in this voyager, while the Universe claims for our efforts!

\section{(c)) EY-NC-ND Licensed to GIRI}

How to cite this article:

Zacharias CR. The High Dilution phenomenon, Yours to Discover. [Editorial]. Int J High Dilution Res [online]. 2009 [cited YYYY Mmm DD]; 8(26): 1-2. Available from: http://www.feg.unesp.br/ ojs/index.php/ijhdr/article/view/331/378. 